\title{
An interplay model for official information and rumor spreading with impulsive effects
}

\author{
Liang'an $\mathrm{HuO}^{1 *}$ (ID and Xiao Li ${ }^{1}$
}

"Correspondence:

huohuolin@yeah.net

${ }^{1}$ Business School, University of

Shanghai for Science and

Technology, Shanghai, China

\section{Springer}

\begin{abstract}
When an emergency event occurs, it inevitably causes some rumors, and the government always issues some official information to control rumors; then the rumors and official information can interact with each other. In this paper, an interplay model between official information and rumor spreading with impulsive effects is proposed to simulate the government strategies in emergency management. By using Floquet theory and comparing methods involving multiple Lyapunov functions, the thresholds for rumor eradication and system permanence are obtained. The influence of the analytical threshold on the stability of a system is verified in a numerical simulation. Finally, some suggestions are provided for the government to eliminate losses causing rumor effect in emergencies.
\end{abstract}

Keywords: Interplay model; Impulsive effects; Official information; Rumors spreading

\section{Introduction}

There will always be arising rumors when a social emergency occurs. Rumors can shape public opinion and affect beliefs, thereby changing individuals' attitude toward social, economic, and political aspects; improper control can easily cause social confusion and losses [1]. Meanwhile the proliferation of rumors will lead to new emergencies and cause cascade reactions. In this sense, the study of spreading of rumors is necessary and of great significance.

Recently, rumor spreading theory has been widely addressed by scholars. Daley and Kendal [2,3] introduced a standard model of rumor spreading, in 1965. In the research field of complex systems, complex network knowledge has been widely used [4]. Zanette used complex network theory to study rumor spreading, and established a rumor spreading model based on a small world network [5]. As the model of rumor spreading has been fully studied and analyzed, scholars also have begun to discuss the various factors affecting rumors [6-8]. The internal and external factors of a social network have a comprehensive effect on rumor spreading, such as node vitality and heterogeneous network spreading environment [9]. In recent years, the study of rumor spreading has undergone various explorations. Aiming at the spreading of rumors online, Zhang et al. proposed an anomaly detection method based on an automatic encoder to detection rumors [10]. Multiple rumors cascading form a network of intensive collaboration, for such networks, the ways to prevent rumors from spreading effectively can borrow groups formed by repetitive ru-

(c) The Author(s) 2019. This article is distributed under the terms of the Creative Commons Attribution 4.0 International License (http://creativecommons.org/licenses/by/4.0/), which permits unrestricted use, distribution, and reproduction in any medium, provided you give appropriate credit to the original author(s) and the source, provide a link to the Creative Commons license, and indicate if changes were made. 
mor spreaders [11]. Based on the dynamic rule of rumor spreading, Han et al. proposed a new SIDR rumor spreading model, considering the psychological factors of "suspicion" and using a full coverage monitoring scheme to prevent and control rumors [12].

The emergency often is accompanied by rumor spreading, which causes social chaos and new derivative accidents. In China, the government always plays the manager role in emergency, and always tends to use official information to dispel the rumors; it will reduce this dampening effect brought about by rumors. Official information is a good factor to guide rumor spreading. Enhancing the enthusiasm of positive news communicators can smoothly guide and restrain rumor spreading, to eliminate a cascade reaction of rumors in emergency events [13-15]. Zhang et al. presented a model describing turnout spreading and emergency development, indicating that rumors propagate at a certain speed [16]. The interaction mechanism between government media can effectively prevent the deterioration of emergency and make rumors play a positive role in the evolution of an emergency [17]. The coupling of official government communication and rumor spreading provides a rational way for limiting the spread of rumors after emergency [18]. The government will adjust the emergency strategies in real time according to the actual situation, issuing public official information about emergency events or refute specific rumors. The government emergency strategies have pulse characteristics with periodical performance [19, 20]. Discrete impulse theory is widely used in exponential synchronization of complex networks with random disturbances strategies [21-23], and this paper add discrete impulse to the differential equation system.

The existing literature lacks interaction research between official information and rumors, in this paper, we consider the characteristics of government emergency strategies and establish an interplay model to simulate the interaction between official information and rumor spreading. Taking government emergency strategies as a pulse adding system, we verified the dynamic behavior of the model by analytical methods and a numerical simulation, and we obtain the thresholds for rumor eradication and system permanence. Finally, we provide some suggestions for the government to eliminate losses caused by rumors in an emergency.

The rest of this paper organized as follows: In Sect. 2, we introduce the proposed model and variables meaning. In Sect. 3, we make preparations by presenting some definitions and three lemmas for the following proofs. In Sect. 4, we study the condition for rumor eradication and system permanence, respectively. In Sect. 5, by numerical simulations we confirm our theoretical results. Finally, a conclusion is given in Sect. 6.

\section{An interplay model for official information and rumor spreading with impulsive effects}

We will reference a functional response function to describe the functional response of official information to relevant rumors [24]. It describes the influence of rumor spreaders to official information disseminators at different stages. With the increase of the number of rumor spreaders, the intensity of rumor spreading first rises and then decreases. Similarly, when rumor spreaders are confronted with government information disseminators (similar to the ignorant who is not familiar with rumors in [24]), the influence of rumors on government information disseminators first increases and then decreases. We have

$$
w(x(t), y(t))=\frac{\beta x(t) y(t)}{1+\alpha x^{2}(t)},
$$


where $x(t)$ represents the number of spreader who hear, believe and spread rumors, $y(t)$ represents the number of disseminators who know and disseminate official information. $\beta$ represents the informed probability of the official information, $\alpha$ represents the psychological effect of the behavioral change of the official information disseminator when the numbers of rumor spreaders change. In fact, the functional response function is the marginal utility of rumors to official information in the interplay process. When the rumor spreader meets the official information disseminator, the former will with a certain probability then transform into the person who disseminates the official information. Owing the inhibition effect or weary effect from the increased rumor spreaders, the transform probability is reduced, influencing the final scope of rumor spread. Therefore, the instant interplay between official information and rumors will reduce the number of rumor spreaders and increase the number of official information disseminators. With fixed $y(t)$, the response function will gradually be increasing when rumor spreader $x(t)$ on a small level, and with the increase of rumor spreaders $x(t), w(x, y)$ will reach the peak value, after that it will be decreasing. This is because of reasons such as the interest of the rumor spreaders and so on, the influence of rumors on government information disseminators first increases and then decreases. If the number of people spreading rumors tends to infinite, the influence of rumors on government information tends to zero.

We assume rumor spreaders $x(t)$ always obeys logistic growth, $r$ is the intrinsic growth coefficient of $x(t)$, and the greatest bearing capacity of society on rumor spreaders is $k$. The functional response function is in Eq. (1), which describes the interplay between official information and rumor spreading, $\gamma$ is the conversion coefficient telling us official information disseminators assimilating the number of the rumor spreaders in unit time. The official information disseminators will lose interest for the already known official information with a rate $d$. We established an impulsive differential system, the function of official information disseminator and rumor spreader, to model the dynamic interplay process of official information and rumors in emergency. The government always tends to use official information to clear up the rumors, it will reduce the negative effects of rumor spreading. We take government strategies of official information as a pulse added to the interplay system in the emergency response process. We divide those strategies into two types: one is refuting specific rumors according to the influence range of rumors, $q$ represents the strength of this strategy $(0 \leq q<1)$. The other is the periodic public official information about emergency events, for which is always assumed that the public official information will release through official channels periodically, $p$ represents the number of new disseminators for each time public official information release $(p \geq 0)$. Then we have an interplay model for official information and rumor spreading with impulsive effects as follows:

$$
\left\{\begin{array}{l}
\frac{d x}{d t}=r x(t)\left[1-\frac{x(t)}{k}\right]-\frac{\beta x(t) y(t)}{1+\alpha x(t)^{2}}, \\
\frac{d y}{d t}=\frac{\gamma \beta x(t) y(t)}{1+\alpha x(t)^{2}}-d y(t), \\
x\left(t^{+}\right)=(1-q) x(t), \\
y\left(t^{+}\right)=y(t)+p,
\end{array}\right\} \quad t \neq n T, n \in N,
$$

where all parameters are positive constants. $T$ is the period of the impulsive effects, $N$ is a positive integer set, $n \in N$. 


\section{Preliminaries}

In this section, we reference the definitions in [25], giving the following notations and three lemmas that will be used for the later sections.

Let $R_{+}=[0, \infty), R_{+}^{2}=\left\{x \in R^{2}: x \geq 0\right\} \Omega=\operatorname{int} R_{+}^{2}, N$ be the set of all non-negative integers. The map $f=\left(f_{1}, f_{2}\right)^{T}$ is defined by the right hand of the first two equations of system (2). Let $V: R_{+} \times R_{+}^{2} \rightarrow R_{+}$, then $V$ is said to belong to class $V_{0}$ if

(1) $V$ is continuous in $(n T,(n+1) T] \times R_{+}^{2}$, and for each $x \in R_{+}^{2}, n \in N$, $\lim _{(t, y) \rightarrow\left(n T^{+}, x\right)} V(t, y)=V\left(n T^{+}, x\right)$ exists.

(2) $V$ is locally Lipschitzian in $x$.

Definition 3.1 $V \in V_{0}$, then, for $(t, x) \in(n T,(n+1) T] \times R_{+}^{2}$, the upper right derivative of $V(t, x)$ with respect to system (2) is defined as

$$
D^{+} V(t, x)=\lim _{h \rightarrow 0^{+}} \sup \frac{1}{h}[V(t+h, x+h f(t, x))-V(t, x)] .
$$

Definition 3.2 System (2) said to be persistent if there exist positive constants $m>0$, $M>0$ (independent of initial value) and a finite time $T_{0}$, such that for each positive solution $(x(t), y(t))$ of system (2) is satisfied $m \leq x(t) \leq M, m \leq y(t) \leq M$, for all $t>T_{0}$.

The solution of system (2) is a piecewise continuous function $x(t): R_{+} \rightarrow R_{+}^{2}, x(t)$ is continuous on $(n T,(n+1) T]$, and $x\left(n T^{+}\right)=\lim _{t \rightarrow n T^{+}} x(t)$ exists. Obviously the smoothness properties of $f$ guarantee the global existence and uniqueness of solutions of system (2) (see $[26,27]$ for details on the fundamental properties of impulsive systems).

It is easy to prove the following lemma.

Lemma 3.1 Let $x(t)$ is a solution of system (2) with $x\left(0^{+}\right) \geq 0$, then $x(t) \geq 0$ for all $t \geq 0$ and further $x(t)>0, t \geq 0$ if $x\left(0^{+}\right)>0$.

We will use the following comparison theorem for later proofs [26].

Lemma 3.2 Suppose $V \in V_{0}$, and assume that

$$
\begin{cases}D^{+} V(t, x) \leq g(t, V(t, x)), & t \neq n T \\ V\left(t, x\left(t^{+}\right)\right) \leq \psi_{n}(V(t, x)), & t=n T\end{cases}
$$

where $g: R_{+} \times R_{+} \rightarrow R$ is continuous in $(n T,(n+1) T] \times R_{+}$. In addition, for $u \in R_{+}, n \in$ $N, \lim _{(t, y) \rightarrow\left(n T^{+}, u\right)} g(t, y)=g\left(n T^{+}, u\right)$ exists $\psi_{n}: R_{+} \rightarrow R_{+}$is non-decreasing. Let $r(t)$ be the maximal solution of the scalar impulsive differential equation,

$$
\left\{\begin{array}{l}
\dot{u}(t)=g(t, u(t)), \quad t \neq n T, \\
u\left(t^{+}\right)=\psi_{n}(u(t)), \quad t=n T, \\
u\left(0^{+}\right)=u_{0},
\end{array}\right.
$$

existing on $[0, \infty)$. Then $V\left(0^{+}, x_{0}\right) \leq u_{0}$ implies that $V(t, x(t)) \leq r(t), t \geq 0$, where $x(t)$ is any solution of system (2). 
Considering the application of comparison theorem in differential equations, we give some basic properties of the following subsystem of system (2):

$$
\left\{\begin{array}{l}
\dot{y}(t)=-d \times y(t), \quad t \neq n T, \\
y\left(t^{+}\right)=y(t)+p, \quad t=n T, \\
y\left(0^{+}\right)=y_{0}
\end{array}\right.
$$

clearly

$$
y^{*}(t)=\frac{p \exp (-d(t-n T))}{1-\exp (-d T)}, \quad t \in(n T,(n+1) T], n \in N
$$

and

$$
y^{*}\left(0^{+}\right)=\frac{p}{1-\exp (-d T)}
$$

the positive periodic solution of system (3):

$$
y(t)=\left(y\left(0^{+}\right)-\frac{p}{1-\exp (-d T)}\right) \exp (-d t)+y^{*}(t)
$$

the solution of system (3) with initial value $y_{0} \geq 0$, where $t \in(n T,(n+1) T], n \in N$. Then we arrive at Lemma 3.3.

Lemma 3.3 Let $y^{*}(t)$ be a positive periodic solution of system (3) and every solution $y(t)$ of system (3) with $y_{0} \geq 0$, we have $\left|y(t) \rightarrow y^{*}(t)\right| \rightarrow 0$ when $t \rightarrow \infty$. Therefore, we obtain the complete expression for the rumor-eradication periodic solution of system (2),

$$
\left(0, y^{*}(t)\right)=\left(0, \frac{p \exp (-d(t-n T))}{1-\exp (-d T)}\right) \quad \text { for } t \in(n T,(n+1) T] .
$$

\section{Rumor eradication and system permanence}

In this section, we will study the condition for rumor eradication and system permanence, respectively. Firstly, we show that all solutions of system (2) ultimately are bounded from Theorem 4.1. By using Floquet theory and the small amplitude perturbation method [28], we obtain the conditions for the local stability of a rumor eradication periodic solution $\left(0, y^{*}(t)\right)$ from Theorem 4.2. We named it the rumor eradication theorem. Based on Definition 3.2, we will show the conditions for the permanence of system (2) from Theorem 4.3. We named it the system permanence theorem.

Theorem 4.1 There exists a constant $M>0$ such that $x(t) \leq M, y(t) \leq M$ for each solution $(x(t), y(t))$ of system (2) with all t large enough.

Proof Suppose $(x(t), y(t))$ is any solution of system (2). Define

$$
V(t)=\gamma x(t)+y(t) .
$$


Then $V \in V_{0}$,

$$
\left\{\begin{array}{l}
D^{+} V(t)+\lambda V(t)=\gamma(r+\lambda) x(t)-\frac{\gamma r}{k} x^{2}(t)+(\lambda-d) y(t), \quad t \neq n T \\
V\left(n t^{+}\right)=V(n t)+p, \quad t=n T
\end{array}\right.
$$

Clearly, the right hand side of system (8) is bounded when $0<\lambda<d$. Select a suitable $\lambda_{0}$ and let $M_{0}$ be the bound. Thus, system (8) leads to

$$
\left\{\begin{array}{l}
D^{+} V(t) \leq M_{0}-\lambda_{0} V(t), \quad t \neq n T \\
V\left(n t^{+}\right)=V(n t)+p, \quad t=n T
\end{array}\right.
$$

By Lemma 3.2, if $t \in(n T,(n+1) T]$, we can get

$$
\begin{aligned}
V(t) \leq & \left(V\left(0^{+}\right)-\frac{M_{0}}{\lambda_{0}}\right) \exp \left(-\lambda_{0} t\right) \\
& +\frac{p\left(1-\exp \left(-n \lambda_{0} T\right)\right)}{\exp \left(\lambda_{0} T\right)-1} \exp \left(\lambda_{0} T\right) \exp \left(-\lambda_{0}(t-n T)\right)+\frac{M_{0}}{\lambda_{0}} .
\end{aligned}
$$

Hence

$$
\lim _{t \rightarrow \infty} V(t) \leq \frac{M_{0}}{\lambda_{0}}+\frac{p \exp \left(\lambda_{0} T\right)}{\exp \left(\lambda_{0} T\right)-1}
$$

Therefore $V(t)$ is ultimately bounded by a constant and there exists a constant $M>0$, such that $x(t) \leq M, y(t) \leq M$ for each solution $(x(t), y(t))$ of system (2) with all $t$ large enough. The proof is completed.

Theorem 4.2 Let $(x(t), y(t))$ be any solution of system (2), if $p>\frac{d}{\beta}\left[r T-\ln \frac{1}{(1-q)}\right]$ then $\left(0, y^{*}(t)\right)$ is said to be locally asymptotically stable.

Proof The locally asymptotical stability of the periodic solution $\left(0, y^{*}(t)\right)$ may be determined by considering the behavior of a small amplitude perturbation of the solution. Define $x(t)=u(t), y(t)=y^{*}(t)+v(t)$. Here $v(t)$ is a small perturbation from system (2), and we have

$$
\left(\begin{array}{l}
u(t) \\
v(t)
\end{array}\right)=\Phi(t)\left(\begin{array}{l}
u(0) \\
v(0)
\end{array}\right), \quad 0 \leq t<T
$$

The matrix solution matrix $\Phi(t)$ satisfies

$$
\Phi(t)=\left(\begin{array}{cc}
\exp \left(t\left(r-\beta y^{*}\right)\right) & 0 \\
\frac{\exp (-d t)\left[\exp \left(t\left(d+r-\beta y^{*}\right)\right)-1\right]}{d+r-\beta y^{*}} \gamma \beta y^{*} & \exp (-d t)
\end{array}\right) .
$$

After adding impulses, system (2) becomes

$$
\left(\begin{array}{l}
u\left(n T^{+}\right) \\
v\left(n T^{+}\right)
\end{array}\right)=\left(\begin{array}{cc}
1-q & 0 \\
0 & 1
\end{array}\right)\left(\begin{array}{l}
u(n T) \\
v(n T)
\end{array}\right)
$$


By the Floquet theory of impulsive differential equations [28], $\left(0, y^{*}(t)\right)$ is locally asymptotically stable if $\left|p_{1}\right|=\exp (-d \times T)$ and $\left|p_{2}\right|=(1-q) \exp \int_{0}^{T}\left(r-\beta y^{*}\right) d t$ the absolute values of both eigenvalues are less than one, thus we obtain the conditions $p>\frac{d}{\beta}\left[r T-\ln \frac{1}{(1-q)}\right]$ for the local stability of the rumor eradication periodic solution.

Theorem 4.3 If $p<\frac{d}{\beta}\left[r T-\ln \frac{1}{(1-q)}\right]$ then system (2) is persistent.

Proof Suppose that $(x(t), y(t))$ is any solution of system (2) with $x(0)>0$. By Theorem 4.1, we have proved that there exists a constant $M>0$, such that $x(t) \leq M, y(t) \leq M$ and $M>k$, for $t$ large enough. Then we will prove that the solution of system (2) has lower bounds to complete the proof of system permanence.

Define

$$
m_{1}=\frac{p \exp (-d T)}{1-\exp (-d T)}-\sigma, \quad \sigma>0
$$

From Lemma 3.2 we can get $\lim _{t \rightarrow \infty} y(t)>m_{1}$, which is to say $y(t)$ has lower bounds. Next we shall find a $m_{2}>0$ so that $x(t) \geq m_{2}$ for $t$ large enough. We will prove this in the following two steps.

Firstly, since $p<\frac{d}{\beta}\left[r T-\ln \frac{1}{(1-q)}\right]$, the solution of system (2) is unstable. We select $0<m_{3}<$ $k$ and $\varepsilon>0$ small enough such that $\delta=\frac{\gamma \beta m_{3}}{1+\alpha m_{3}^{2}}<d, \sigma=r T-\frac{r}{k} m_{3} T-\beta \frac{p}{d-\delta}-\varepsilon_{1} T>0$. Now we assume that there exists $t_{1} \in(0, \infty)$ such that $x\left(t_{1}\right)<m_{3}$. We call this assumption one.

Thus, we can get $\dot{y}(t) \leq y(t)(\delta-d)$. Considering the impulsive differential equation.

$$
\left\{\begin{array}{l}
\dot{u}(t)=u(t)(\delta-d), \quad t \neq n T \\
u\left(t^{+}\right)=u(t)+p, \quad t=n T \\
u\left(0^{+}\right)=y_{0}>0
\end{array}\right.
$$

By Lemmas 3.2 and 3.3, we have $y(t) \leq u(t)$ and $u(t) \rightarrow \bar{u}(t), t \rightarrow \infty$. Here

$$
\bar{u}(t)=\frac{p \exp [(\delta-d)(t-n T)]}{1-\exp [(\delta-d) T]}, \quad t \in(n T,(n+1) T]
$$

exists $T_{1}>0$, when $t>T_{1}$,

$$
y(t) \leq u(t) \leq \bar{u}(t)+\varepsilon
$$

and

$$
\dot{x}(t) \geq x(t)\left[r-\frac{r}{k} m_{3}-\beta\left(\bar{u}(t)+\varepsilon_{1}\right)\right]
$$

let $N_{1} \in N$ and $N_{1} T>T_{1}, n \leq N_{1}$, we will have

$$
\left\{\begin{array}{l}
\dot{x}(t) \geq x(t)\left[r-\frac{r}{k} m_{3}-\beta\left(\bar{u}(t)+\varepsilon_{1}\right)\right], \quad t \neq n T, \\
x\left(t^{+}\right)=(1-q) x(t), \quad t=n T
\end{array}\right.
$$


moreover, we can obtain the following result:

$$
\begin{aligned}
x((n+1) T) & \geq x(n T) \exp \left(\int_{n T}^{(n+1) T}\left[r-\frac{r}{k} m_{3}-\beta\left(\bar{u}(t)+\varepsilon_{1}\right)\right] d t\right) \\
& =x(n T) \exp (\sigma) .
\end{aligned}
$$

Then $x\left(\left(N_{1}+l\right) T\right) \geq x\left(N_{1} T^{+}\right) \exp (l \sigma) \rightarrow \infty$ as $l \rightarrow \infty$, which is a contradiction to Theorem 4.1. Therefore, the first assumption is not true. Hence there exists a $t_{1}>0$ such that $x\left(t_{1}\right) \geq m_{3}$.

Secondly, we only need to consider those solutions that leave the region $R=\left\{X(t) \in R_{+}^{2}\right.$ : $\left.x(t)<m_{3}\right\}$ moreover, reenter again. Let $t^{*}=\inf _{t \geq t_{1}}\left\{x(t)<m_{3}\right\}$, then we have two cases for discussion.

Case 1: if the $t^{*}$ is an impulsive point, there exists $n_{0} \in N, t^{*}=n_{0} T, \varepsilon^{*}>0$ small enough. We have $t_{*}=t^{*}-\varepsilon^{*}$, so $t_{*}$ is a non-impulsive point and $x\left(t_{*}\right) \geq m_{3}$.

Case 2: if the $t^{*}$ is a non-impulsive point, $x(t) \geq m_{3}$ on $\left[t_{1}, t^{*}\right)$ and $x\left(t^{*}\right)=m_{3}$.

Let us assume $t^{*} \in\left[n_{1},\left(n_{1}+1\right) T\right), n_{1} \in N$, is a non-impulsive point. We call this assumption two. Choose $n_{2}, n_{3} \in N$, such that

$$
n_{2} T>T_{2}=\frac{\ln \left(\frac{\varepsilon_{1}}{M+p}\right)}{-d+\delta}, \quad \exp \left(\left(n_{2}+1\right) \sigma_{1} T\right) \exp \left(n_{3} \sigma\right)>1
$$

Here $\sigma_{1}=r-\frac{r}{k} m_{3}-\beta M<0$. Let $\bar{T}=\left(n_{2}+n_{3}\right) T$, there exists $t_{2} \in\left(\left(n_{1}+1\right) T,\left(n_{1}+1\right) T+\bar{T}\right)$, such that $x\left(t_{2}\right) \geq m_{3}$, now suppose that $t \in\left(\left(n_{1}+1\right) T,\left(n_{1}+1\right) T+\bar{T}\right)$, satisfy $x(t)<m_{3}$ we call this assumption three. Considering

$$
\left\{\begin{array}{l}
\dot{u}(t)=u(t)(\delta-d), \quad t \neq n T, \\
u\left(t^{+}\right)=u(t)+p, \quad t=n T, \\
u\left(0^{+}\right)=y_{0}>0
\end{array}\right.
$$

let $y\left(\left(n_{1}+1\right) T^{+}\right)=u\left(\left(n_{1}+1\right) T^{+}\right)$and we have

$$
u(t)=\left(u\left(\left(n_{1}+1\right) T^{+}\right)-\frac{p}{1-\exp ((-d+\delta) T)}\right) \exp (-d+\delta)\left(t-\left(n_{1}+1\right) T\right)+\bar{u}(t)
$$

where $t \in(n T,(n+1) T), n_{1}+1 \leq n \leq n_{1}+1+n_{2}+n_{3}$.

So, when $\left(n_{1}+1+n_{2}\right) T \leq t \leq\left(n_{1}+1\right) T+\bar{T}$, is satisfied then

$$
|u(t)-\bar{u}(t)|<(M+q) \exp (-d+\delta)<\varepsilon_{1}
$$

and

$$
y(t) \leq u(t) \leq \bar{u}(t)+\varepsilon .
$$

as in the first step, when $t \in\left[\left(n_{1}+1+n_{2}\right) T,\left(n_{1}+1\right) T+\bar{T}\right]$ we have

$$
x\left[\left(n_{1}+1+n_{2}+n_{3}\right) T\right] \geq x\left[\left(n_{1}+1+n_{2}\right) T\right] \exp \left(n_{3} \sigma\right)
$$


the first equation of system (2) gives

$$
\dot{x}(t) \geq x(t)\left[r-\frac{r}{k} m_{3}-\beta M\right]=\sigma_{1} x(t)
$$

integrating Eq. (25) on $\left[t^{*},\left(n_{1}+1+n_{2}\right) T\right]$, we can get

$$
x\left[\left(n_{1}+1+n_{2}\right) T\right] \geq m_{3} \exp \left(\sigma_{1}\left(n_{2}+1\right) T\right)
$$

thus, from Eqs. (24) and (26), we have

$$
x\left[\left(n_{1}+1+n_{2}+n_{3}\right) T\right] \geq m_{3} \exp \left(\sigma_{1}\left(n_{2}+1\right) T\right) \exp \left(n_{3} \sigma\right)>m_{3}
$$

which is a contradiction to assumption three. Therefore, assumption three is not true. Thus for $t \in\left(n_{1} T,\left(n_{1}+1\right) T+\bar{T}\right]$ we get $x(t) \geq m_{3}$. And assumption two is really true, $t^{*}$ is a non-impulsive point, $x\left(t^{*}\right)=m_{3}$.

Let $\bar{t}=\inf _{t \geq t^{*}}\left\{x(t) \geq m_{3}\right\}$, then $x(\bar{t}) \geq m_{3}$, for $t \in\left[t^{*}, \bar{t}\right)$, we have

$$
x(t) \geq x\left(t^{*}\right) \exp \left(\sigma_{1}\left(t-t^{*}\right)\right) \geq m_{3} \exp \left(\sigma_{1}\left(1+n_{2}+n_{3}\right) T\right) \triangleq m_{2} .
$$

For $t>\bar{t}$, the same arguments hold since $x(\bar{t}) \geq m_{3}$. Hence $x(t) \geq m_{2}$, for all $t>t_{1}$. That is, for $t \rightarrow \infty, x(t)$ has lower bounds. The proof is completed. In summary, for $p<\frac{d}{\beta}[r T-$ $\left.\ln \frac{1}{(1-q)}\right]$ system (2) is persistent.

\section{Numerical simulations}

From Theorem 4.2 and Theorem 4.3, we know that the impulsive differential system describes the interplay between official information and rumor spreading in emergency, and there exist two states: rumor eradication and system permanence. We see that the rumor eradication periodic solution $\left(0, y^{*}(t)\right)$ is locally asymptotically stable if the condition $p>p_{\text {thr }}=\frac{d}{\beta}\left[r T-\ln \frac{1}{(1-q)}\right]$, is satisfied and system (2) is persistent if $p<p_{t h r}$. Moreover, we also get an inference about the rumor eradication and system permanence as follows.

Inference 5.1 Let $(x(t), y(t))$ be any solution of system (2), if the condition $q>q_{t h r}=1-$ $\exp \left(\frac{p \beta}{d}-r T\right)$ is satisfied, then $\left(0, y^{*}(t)\right)$ is said to be locally asymptotically stable. If $q<$ $q_{t h r}$, then system (2) is persistent.

The two government strategies we mentioned above are: firstly, timely release of public official information on emergency events, through government bulletins, government websites, press conferences, newspapers, radio, television and other news media to make the masses aware of the event and avoid panic. Secondly, according to the influence range of various rumors, refuting the specific rumors through government information channels in a timely manner. As is well known, the corresponding continuous system (2) cannot be solved explicitly and is not an infinite system. So we have to study the numerical simulations of system (2), and consider the following set of parameters to our analysis:

$$
\begin{array}{ll}
r=0.95, & \beta=0.04, \quad \gamma=0.5, \quad d=0.2, \\
\alpha=0.01, \quad k=1000, \quad T=3 . &
\end{array}
$$




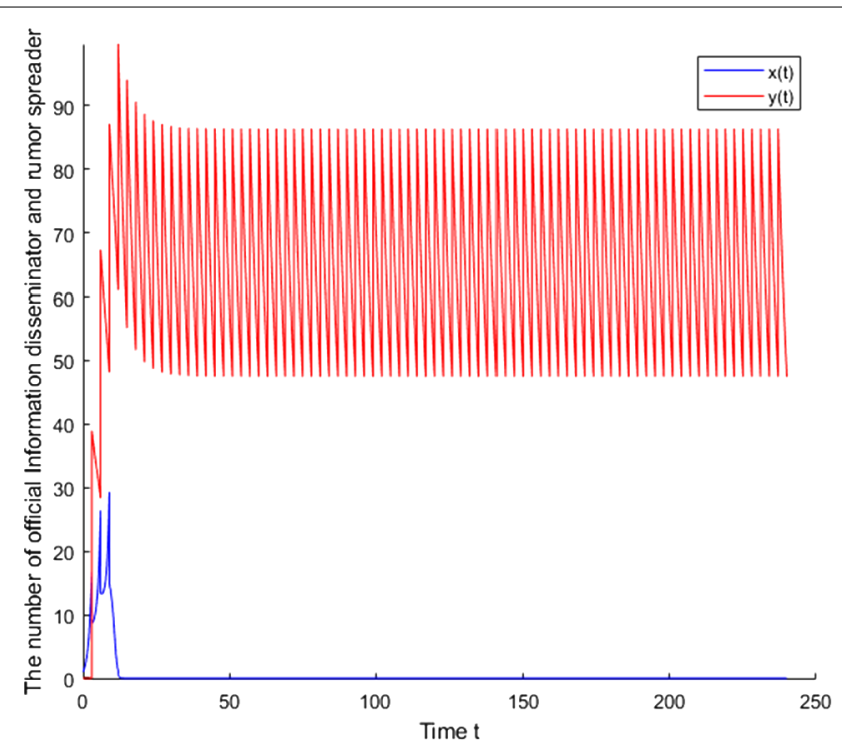

Figure 1 Time-series of the spreader $x$ for eradication and the disseminator $y$ for oscillation

According to Theorems 4.2-4.3 and Inference 5.1, we get the threshold value of the public official information pulse $p$ and the threshold values for the pulse strength $q$ of refuting specific rumors. If $p>p_{t h r}$ or $q>q_{t h r}$, the periodic solution $\left(0, y^{*}(t)\right)$ is locally asymptotically stable. A typical rumor eradication periodic solution is obtained through simulation, and we find that the variable $y(t)$ oscillates in a stable cycle, whereas the number of rumor spreaders $x(t)$ drops rapidly to zero (Fig. 1 ). The state of rumor eradication is that over time, the validity of the official information is oscillating in a stable limit cycle, and the negative social impact of rumors tends to disappear. If $p<p_{t h r}$ or $q<q_{t h r}$ the system is persistent, that is, rumors can be spread among all people, and the official information disseminator is far lower than the rumor spreaders (Fig. 2). The phase portrait (T-periodic solution) of $x(t), y(t)$ shows $x$ and $y$ can coexist on a positive periodic solution, an early shock eventually forms a limit cycle (Fig. 3). We say the system persistent if we have validity of official information and a negative influence of rumors, oscillation tends to a stable limit cycle, continuing by oscillation in a quasi-periodic or periodic period.

In addition, we study the effects of two kinds of pulses to the system, in the manner of control variate. Firstly fixing the parameter $q=0.5$, we get the threshold of $p$ by calculating the system parameters: $p_{t h r}=39$. Moreover, setting different $p$ values, we found that, if $p>p_{t h r}$ is satisfied, and the rumor spreaders $x(t)$ will tend to zero. The larger the value of $p$ is, the faster $x(t)$ tends to zero, the smaller the influence range of rumor spreading (Fig. 4). At the same time, the official information disseminator $y(t)$ is directly proportional to the public official information pulse about the emergency. The larger the value of $p$ is, the more people on the impulse period node who get official information (Fig. 5). This is because the government has intensified its propaganda of official information, and more and more people have been informed of official information, thus affecting the spread of rumors. As long as the intensity is greater than a certain threshold, the rumors will disappear in the end.

When $p<p_{t h r}$, both official information and rumors exist in society. The smaller the value of $p$, the quicker a rumor spreader $x(t)$ reaches the periodic oscillation (Fig. 6). On the 


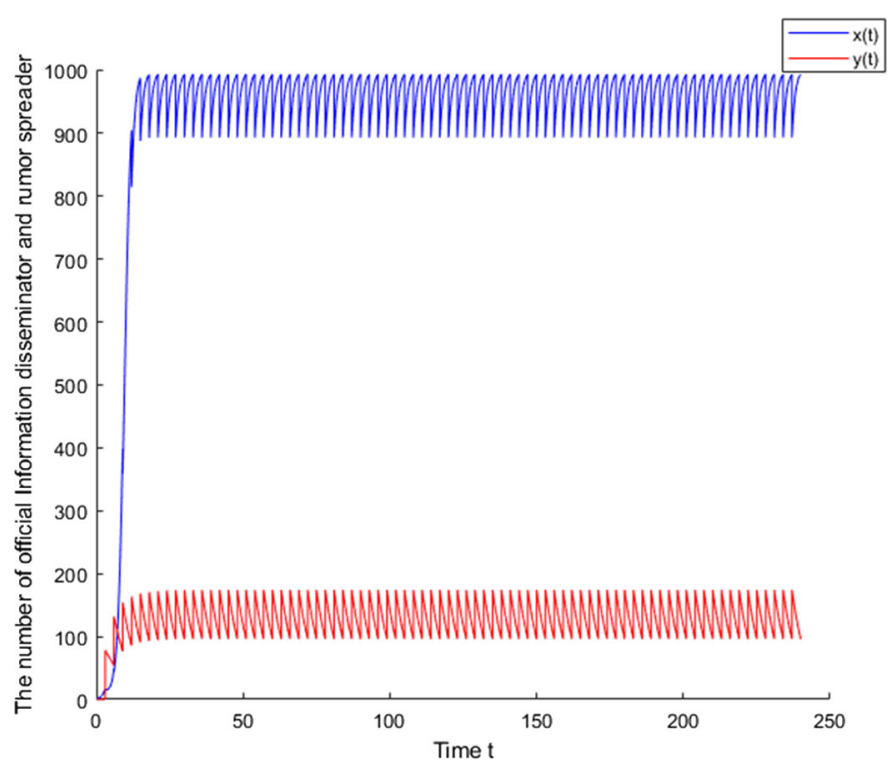

Figure 2 Time-series of the spreader $x$ and the disseminator $y$ for permanence

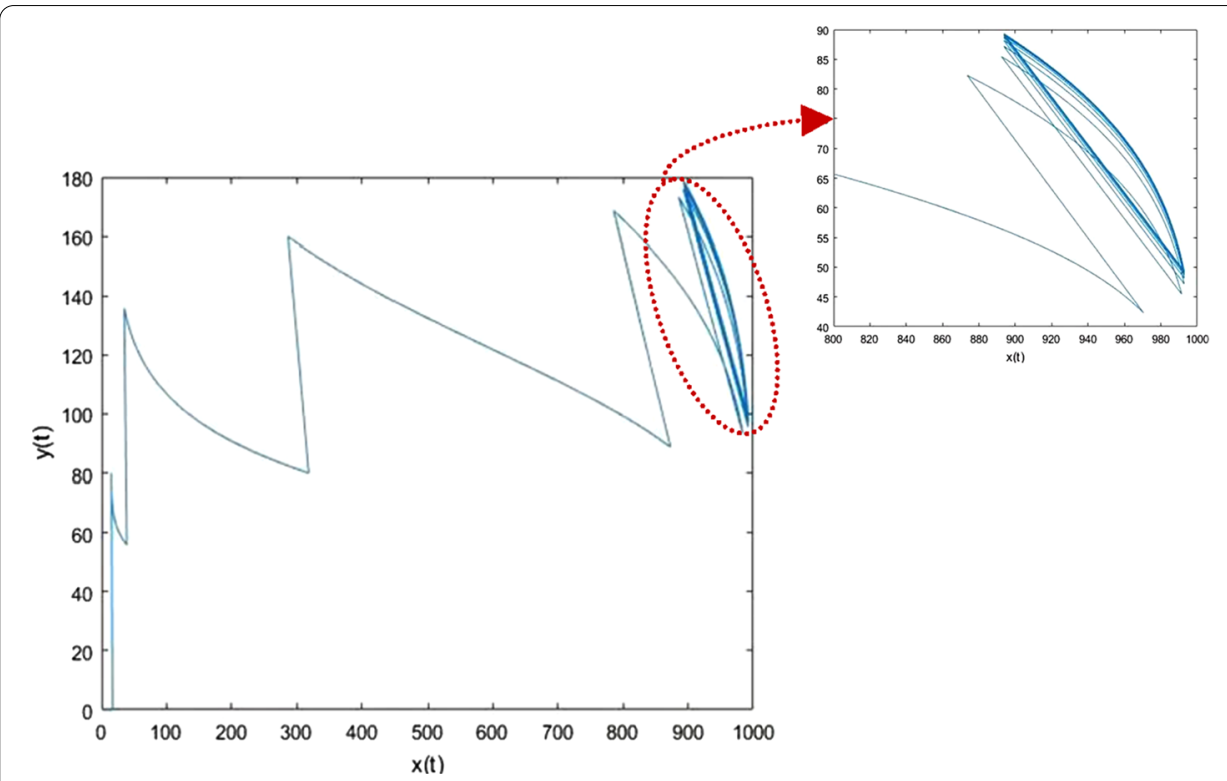

Figure 3 Phase portrait (T-periodic solution) of the spreader and the disseminator population of system (2)

contrary, $p$ value has a significant effect on official information disseminator. The larger the value of $p$, the more the official information disseminator is on the impulse period node. The smaller the value of $p$, the fewer official information disseminators on the impulse period node, which disseminator will oscillate between smaller values, but will not tend to zero (Fig. 7).

In view of the situation that the value of $p$ mainly affects the official emergency validity, we consider that if the negative impact of rumor spreading is still below the warning line (referring to $p<p_{t h r}$ ), we just need to make sure that the social loss caused by rumor 


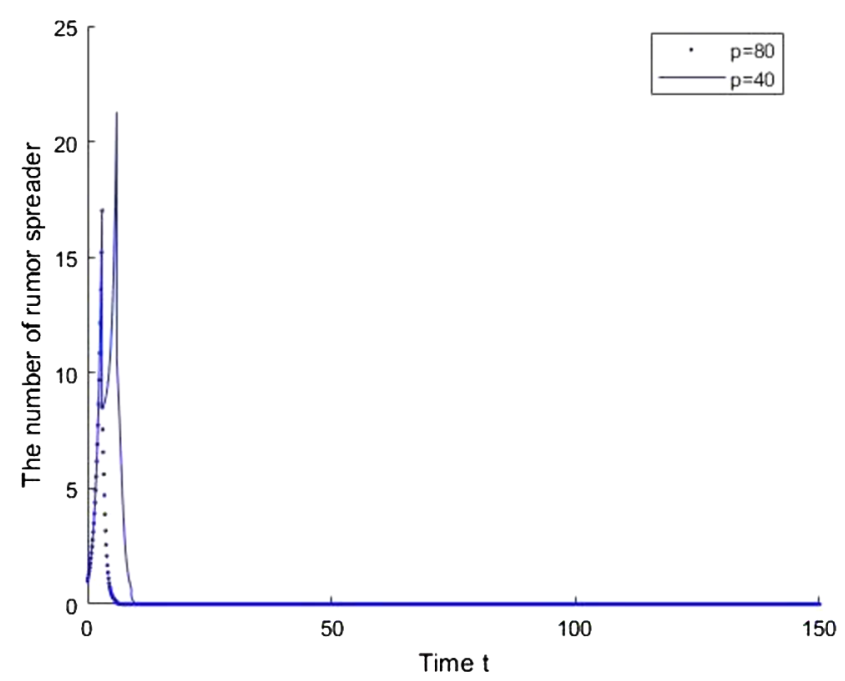

Figure 4 When $p>p_{\text {thr }}$, the evolution graphs with different $p$ of $x(t)$ both tend to 0

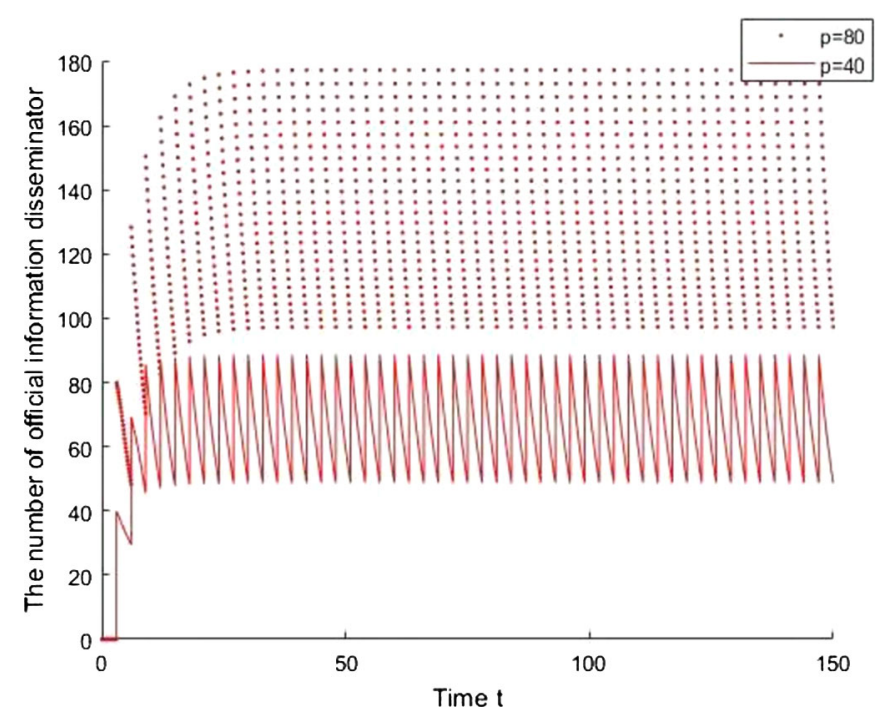

Figure 5 When $p>p_{\text {thr }}$, the evolution graph with different $p$ of $y(t)$ are in oscillation

spreading will not worsen further. Our conclusion is: strengthen the official emergency response, pay attention to the real-time dynamics of emergency, do a timely release of public official information on events, and bring the emergency into the field of emergency development.

Secondly we will talk about pulse $q$ which corresponds with the influence range of different rumors refuting major rumors. Its main way is to choose reliable information in disseminated channels and explain main rumors scientifically and reasonably, so as to reduce the number of rumor spreaders on the impulse periodic nodes. The refuting specific rumor strength pulse $q$ mainly affects the rumor spreaders. We fix the parameter $p=40$ to calculate the threshold of $q$ and get $q_{t h r}=0.494$, then set different $q$ values. When $q>q_{t h r}$, the rumor spreaders $x(t)$ finally tend to zero. The larger $q$, the smaller the range of rumor 


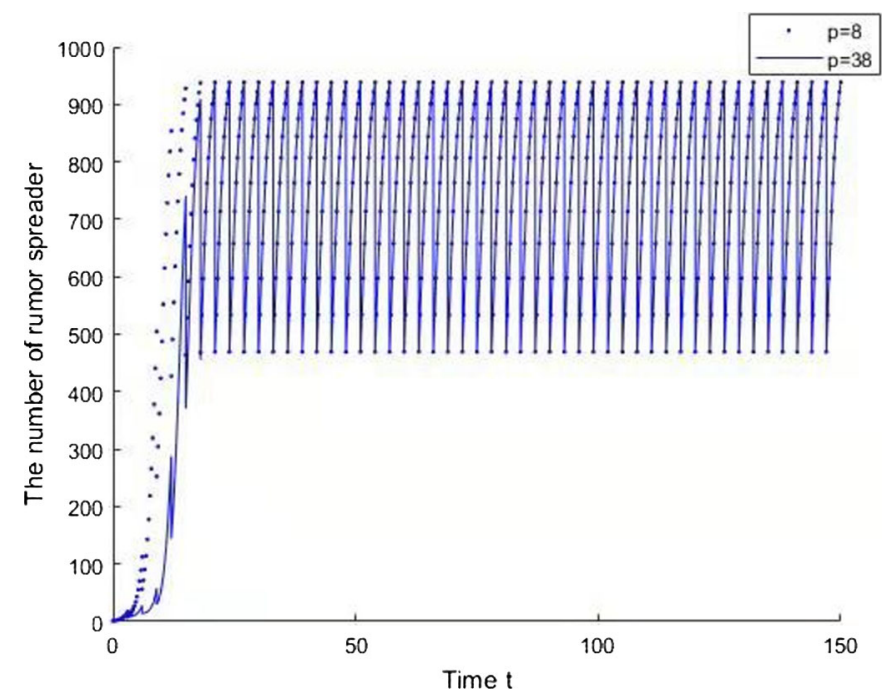

Figure 6 When $p<p_{\text {thr }}$, the evolution graph with different $p$ of $x(t)$ show a periodic solution

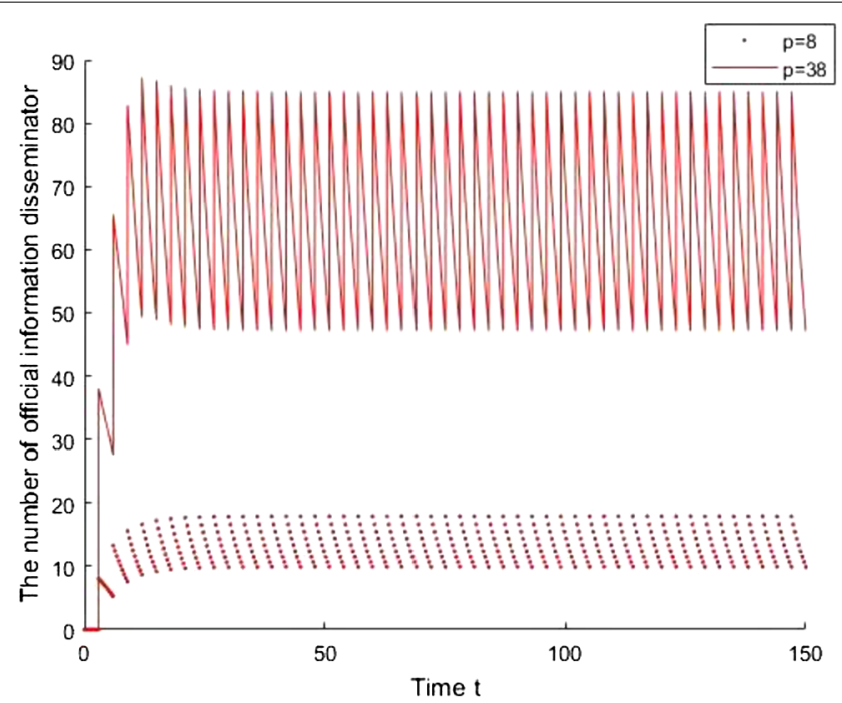

Figure 7 When $p<p_{\text {thr }}$, the evolution graph with different $p$ of $y(t)$ show a periodic solution

spreading, the faster the rumor spreaders tend to zero (Fig. 8). Compared with the different values of $q$, all the official information disseminators $y(t)$ oscillate on the same level of positive periodic solution (Fig. 9).

When $q<q_{t h r}$, the smaller value of $q$ will make the rumor spreaders approach a constant value which means rumors break out and everyone knows rumors. The smaller the value of $q$, the more rumor spreaders on the impulse period node, we say that the rumor spreader is inversely proportional to the refuting specific rumors strength pulse $q$ (Fig. 10). With different $q$ values, the official information disseminator $y(t)$ oscillates on the same level of positive periodic solution (Fig. 11).

Compared with the $p$ impulse, the impact of the $q$ impulse on the system is mainly on the dynamic process of rumor spreading. When there is a response to emergency events, with 


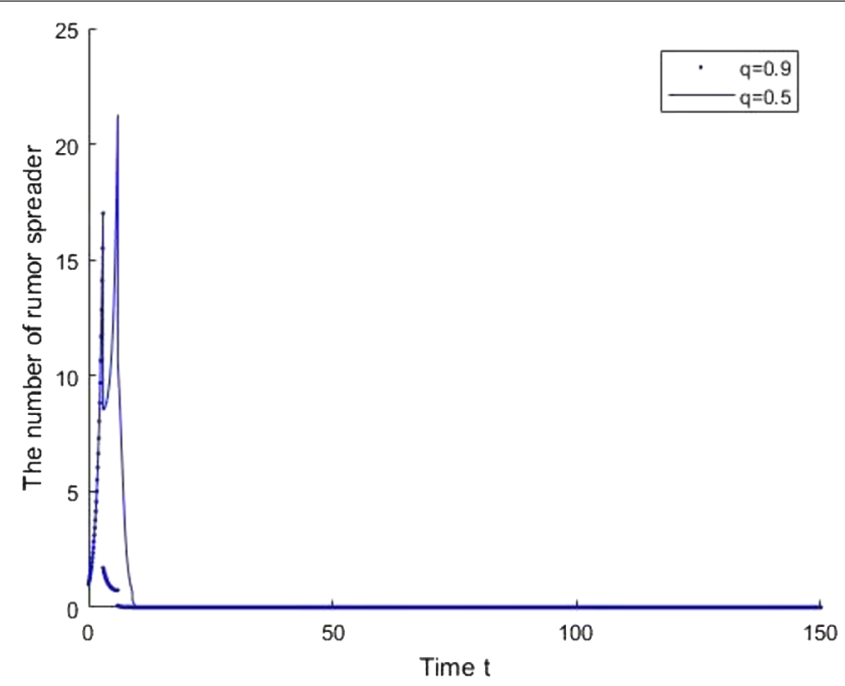

Figure 8 When $q>q_{\text {thr }}$, the evolution graph with different $q$ of $x(t)$ both tend to 0

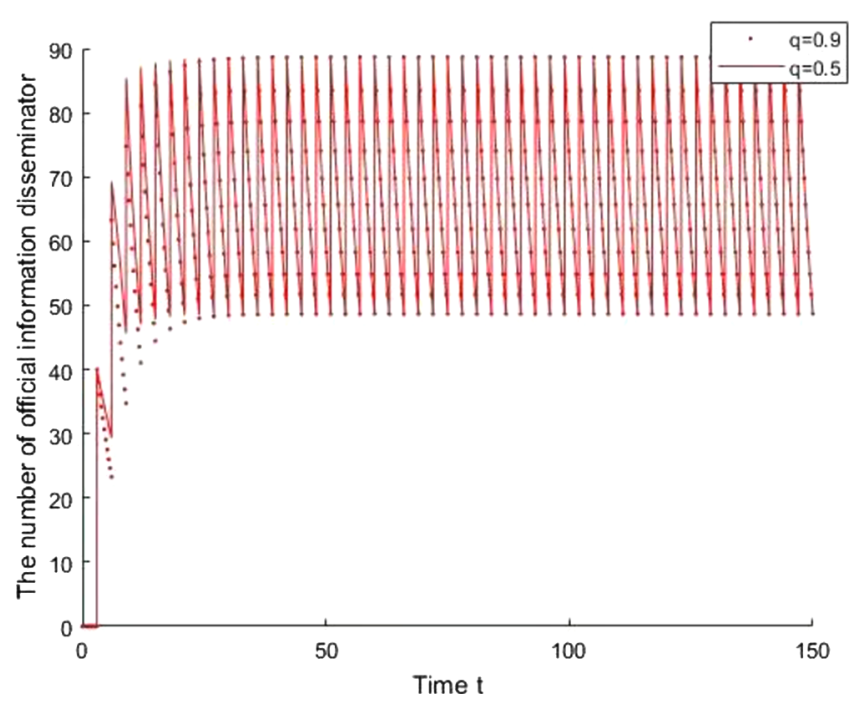

Figure 9 When $q>q_{\text {thr }}$, the evolution graph with different $q$ of $y(t)$ are in oscillation

regard to the serious negative impact on society by the rumor spreading, the government should consider to stop to report the public official information temporary (i.e. pulse $p$ can be zero), make every effort to suppress all kinds of rumors, strengthen the $q$ pulse strength of refuting specific rumors, and make the rumor spreaders tend to zero quickly in the system.

\section{Conclusions}

By considering the characteristics of government emergency strategies, an interplay model between official information and rumor spreading was established. There are two kinds of government strategies as pulse effects are added to the system, one is refuting specific rumors, and the other is the periodic public official information on emergency events; both strategies can eliminate rumors under certain conditions. Otherwise, rumors are in 


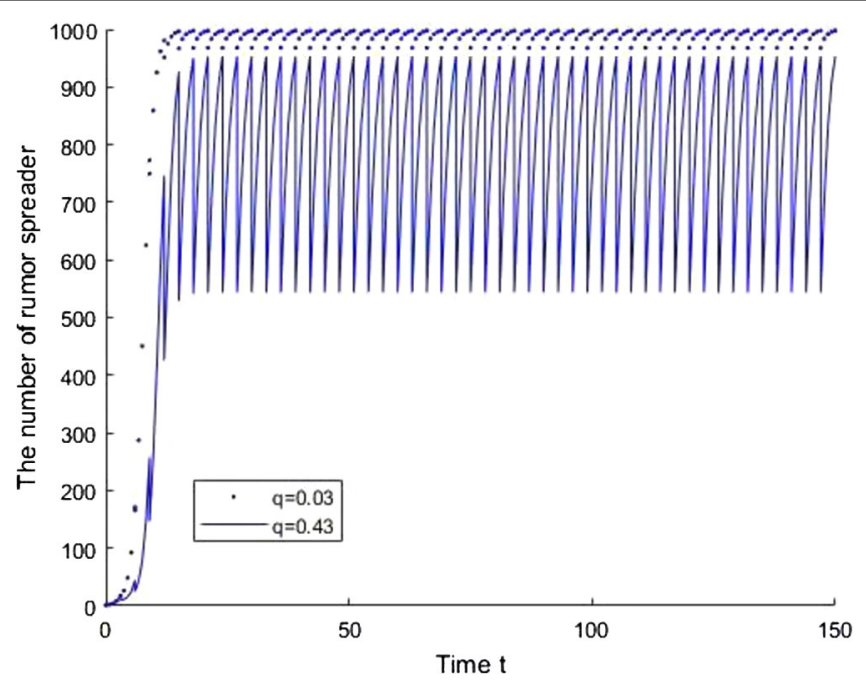

Figure 10 When $q<q_{\text {thr }}$, the evolution graph with different $q$ of $x(t)$ are in a periodic solution

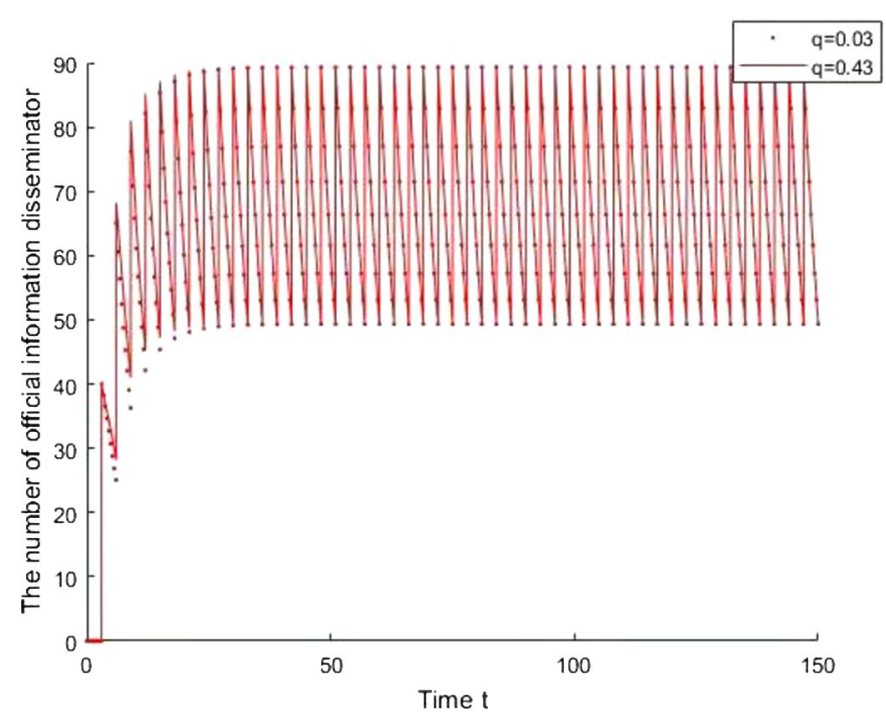

Figure 11 When $q<q_{\text {thr }}$, the evolution graph with different $q$ of $y(t)$ are in a periodic solution

flood and the system is a persistent system, which may generate cascade reactions and trigger new emergencies. The numerical simulation confirmed our conclusion, that is to say, to eliminate social losses caused by rumors and calm down emergency effectively, the government can increasingly issue public official information of events, or increase the strength of refuting specific rumors according to the influence range of rumors timely.

\section{Acknowledgements}

The authors are very grateful to the anonymous referees for their valuable comments and suggestions, helping them to improve the quality of this paper.

\section{Funding}

This work partially supported by the Project of Humanity and Social Science foundation of University of Shanghai Science and Technology (SK17ZD02, SK18ZD03), the Project of Fundamental Research Funds for the Central Universities (2017ECNV-KXK016), and the National Natural Science Foundation of China $(71774111,61702331,71303157)$. 


\section{Competing interests}

The authors declare that they have no competing interests.

\section{Authors' contributions}

LAH conceived of the study, proposed the project, helped to draft the manuscript. XL carried out the computations in the proof, and drafted the manuscript. All authors read and approved the final manuscript.

\section{Publisher's Note}

Springer Nature remains neutral with regard to jurisdictional claims in published maps and institutional affiliations.

Received: 17 January 2019 Accepted: 14 April 2019 Published online: 30 April 2019

\section{References}

1. Ndii, M.Z., Carnia, E., Supriatna, A.K.: Mathematical models for the spread of rumors: a review. In: Issues and Trends in Interdisciplinary Behavior and Social Science, pp. 65-73. CRC Press, Boca Raton (2018)

2. Daley, D.J., Kendall, D.G.: Stochastic rumours. IMA J. Appl. Math. 1(1), 42-55 (1965)

3. Daley, D.J., Gani, J.: Epidemic Modelling: An Introduction. Cambridge University Press, Cambridge (2001)

4. Nekovee, M., Moreno, Y., Bianconi, G., et al.: Theory of rumor spreading in complex social networks. Phys. A, Stat. Mech. Appl. 374(1), 457-470 (2007)

5. Zanette, D.H.: Criticality of rumor propagation on small world networks. arXiv preprint (2001). arXiv:cond-mat/0109049, https://doi.org/10.1103/physreve.65.041908

6. Hu, Y., Pan, Q., Hou, W., et al.: Rumor spreading model with the different attitudes towards rumors. Phys. A, Stat. Mech. Appl. 502, 331-344 (2018)

7. Liu, W., Wu, X., Yang, W., et al.: Modeling cyber rumor spreading over mobile social networks: a compartment approach. Appl. Math. Comput. 343, 214-229 (2019)

8. Dong, S., Fan, F.H., Huang, Y.C.: Studies on the population dynamics of a rumor-spreading model in online social networks. Phys. A, Stat. Mech. Appl. 492, 10-20 (2018)

9. Huo, L., Cheng, Y., Liu, C., et al.: Dynamic analysis of rumor spreading model for considering active network nodes and nonlinear spreading rate. Phys. A, Stat. Mech. Appl. 506, 24-35 (2018)

10. Zhang, Y., Chen, W., Yeo, C.K., et al.: Detecting rumors on online social networks using multi-layer autoencoder. In: Technology \& Engineering Management Conference (TEMSCON), pp. 437-441. IEEE, New York (2017)

11. Li, D., Gao, J., Zhao, J., et al.: Repetitive users network emerges from multiple rumor cascades. arXiv preprint (2018). arXiv:1804.05711

12. Han, Q., Miao, F., Fan, W.: Rumor spreading and monitoring deployment in online social networks. In: Communication Technology (ICCT), 2017 IEEE 17th International Conference on, pp. 1347-1351. IEEE, New York (2017)

13. Huo, L., Huang, P., Fang, X.: An interplay model for authorities' actions and rumor spreading in emergency event. Phys. A, Stat. Mech. Appl. 390(20), 3267-3274 (2011)

14. Luo, Y., Ma, J.: The influence of positive news on rumor spreading in social networks with scale-free characteristics. Int. J. Mod. Phys. C 29(9), 1850078 (2018)

15. Hughes, A.L., Palen, L.: The evolving role of the public information officer: an examination of social media in emergency management. J. Homel. Secur. Emerg. Manag. 9(1) (2012). https://doi.org/10.1515/1547-7355.1976

16. Zhang, Z., Zhang, Z.: An interplay model for rumor spreading and emergency development. Phys. A, Stat. Mech. Appl. 388(19), 4159-4166 (2009)

17. Zhao, L., Wang, Q., Cheng, J., et al.: The impact of authorities' media and rumor dissemination on the evolution of emergency. Phys. A, Stat. Mech. Appl. 391(15), 3978-3987 (2012)

18. Xu, J., Zhang, M., Ni, J.: A coupled model for government communication and rumor spreading in emergencies. Adv. Differ. Equ. 2016(1), 208 (2016)

19. Milman, V.D., Myshkis, A.D.: On the stability of motion in the presence of impulses. Sib. Math. J. 1(2), $233-237$ (1960)

20. Myshkis, A.D., Samoilenko, A.M.: Systems with impulses in prescribed moments of the time. Mat. Sb. 74(2), 202-208 (1967)

21. Agur, Z., Cojocaru, L., Mazor, G., et al.: Pulse mass measles vaccination across age cohorts. Proc. Natl. Acad. Sci. USA 90(24), 11698-11702 (1993)

22. Zhang, Q., Chen, G., Wan, L.: Exponential synchronization of discrete-time impulsive dynamical networks with time-varying delays and stochastic disturbances. Neurocomputing 309, 62-69 (2018)

23. Zhao, Y., Zhang, L., Tang, M., et al.: Bounded confidence opinion dynamics with opinion leaders and environmental noises. Comput. Oper. Res. 74, 205-213 (2016)

24. Huo, L., Lin, T., Huang, P.: Dynamical behavior of a rumor transmission model with psychological effect in emergency event. Abstr. Appl. Anal. 2013, Article ID 282394 (2013)

25. Liu, Z., Tan, R.: Impulsive harvesting and stocking in a Monod-Haldane functional response predator-prey system. Chaos Solitons Fractals 34(2), 454-464 (2007)

26. Lakshmikantham, V., Simeonov, P.S.: Theory of Impulsive Differential Equations. World Scientific, Singapore (1989)

27. Simeonov, P.S.: Impulsive Differential Equations: Asymptotic Properties of the Solutions. World Scientific, Singapore (1995)

28. Bainov, D., Simeonov, P.: Impulsive Differential Equations: Periodic Solutions and Applications. CRC Press, Boca Raton (1993) 